\title{
ON A PROBLEM OF M. A. ZORN
}

PIERRE LELONG

1. Introduction. M. A. Zorn has proved $[1]^{1}$ the following result: If every substitution

$$
S_{a b}: \quad x=a t, \quad y=b t,
$$

in which $a$ and $b$ are complex numbers, transforms the formal development $F=\sum_{i, j} a_{i j} x^{i} y^{j}$ into a power series $F_{a, b}(t)$ with a nonvanishing radius of convergence, the series $\bar{F}=\sum_{i, j}\left|a_{i j} x^{i} y^{j}\right|$ converges for sufficiently small $|x|$ and $|y|$.

The conclusion is, in other terms: $F$ is the Taylor series of a function of two complex variables $x, y$ which is analytic at the point $(x=0, y=0)$.

M. A. Zorn suggested, and Rimhak Ree [2] proved recently, that it is sufficient to consider, in the hypothesis, the substitutions $S_{a b}$ with $a$ and $b$ real.

This result is however incomplete: we are here resolving the following problem: what are the minimal conditions which must be imposed on a class $\left[S_{a b}\right]$ of substitutions (1.1) transforming $F$ into a convergent power series $F_{a, b}(t)$, in order that the preceding conclusion hold for any given formal development $F$ ?

It is convenient, for our purpose, to introduce the following definition.

Definition. The class $\left[S_{a, b}\right]$ of substitutions (1.1) is called normal if the convergence of the power series $F_{a, b}(t)$ for every $S_{a b} \in\left[S_{a, b}\right] \mathrm{im}$ plies for any given formal development $F$ to be the Taylor series of an analytic function of $x, y$.

The following remarks will be useful: if $\lambda$ is a complex number different from zero, and $\alpha=\lambda a, \beta=\lambda b$, then the series $F_{\alpha \beta}(t)$ and $F_{a b}(t)$ are simultaneously convergent or divergent; the substitution $S_{00}$ is degenerate and we may suppose that it is excluded from $\left[S_{a, b}\right]$.

We consider therefore the correspondences

$$
\left[S_{a, b}\right] \rightarrow E(a, b) \rightarrow E(\beta) .
$$

$E(a, b)$ is the image set of $\left[S_{a, b}\right]$ in the complex projective space with homogeneous coordinates $a, b$; the point of coordinates $a, b$ belongs to $E(a, b)$ if, and only if, $S_{a, b}$ belongs to $\left[S_{a, b}\right]$ and if $(a, b)$ is different from the pair $(0,0)$.

$E(\beta)$ is the image set of $\left[S_{a, b}\right]$ in the whole complex plane by $\beta=b / a$.

Received by the editors October 24, 1949.

1 Numbers in brackets refer to the bibliography at the end of the paper. 
For brevity's sake, we shall say that the set $E$ is an $F_{\sigma}$ if $E$ is the sum of an enumerable set of bounded and closed sets.

Then the solution of the above problem is given by the following theorem.

TheOREM 1. The class $\left[S_{a, b}\right]$ of substitutions (1.1) is a normal one if, and only if, the image set $E(\beta)$ is not contained in an $F_{\sigma}$ of vanishing capacity.

The non-normal classes $\left[S_{a, b}\right]$ are therefore in correspondence with the $F_{\sigma}$ of vanishing capacity and their subsets in the complex plane of the variable $\beta=b / a$.

Theorem 1 is a consequence of two different results.

Theorem 1a (Generalisation of M. A. Zorn's Theorem). If every substitution (1.1) of a class $\left[S_{a, b}\right]$ corresponding by (1.2) to an image set $E(\beta)$ of positive capacity transforms the formal development $F=\sum_{i, j} a_{i j} x^{i} y^{j}$ into a power series $F_{a b}(t)$ with a nonvanishing radius of convergence, then $F$ is analytic in $x, y$, and $\bar{F}=\sum_{i, j}\left|a_{i j} x^{i} y^{j}\right|$ converges for sufficiently small $|x|,|y|$.

The same result is true, more generally, if $E(\beta)$ cannot be included in an $F_{\sigma}$ of vanishing capacity.

THEOREM $1_{b}$. If the image set $E(\beta)$ of the given class $\left[S_{a, b}\right]$ of substitutions (1.1) is contained in an $F_{\sigma}$ of vanishing capacity, then there exists a development $F=\sum_{i, j} a_{i j} x^{i} y^{j}$ with the following properties:

(a) Every substitution $S_{a b} \in\left[S_{a, b}\right]$ transforms $F$ into a power series $F_{a b}(t)$ with a vanishing radius of convergence.

(b) $\bar{F}=\sum_{i, j}\left|a_{i j} x^{i} y j\right|$ diverges except for $x=y=0$.

Theorem $1_{b}$ shows that a complete solution of M. A. Zorn's problem could not be obtained without making use of the notion of capacity of sets, or, at least, of the class of sets of vanishing capacity in the complex plane.

2. For the convenience of the reader, we shall give a brief account of some results of the theory of potential and subharmonic ${ }^{2}$ functions, which are useful in this paper. It is sufficient, for our purpose, to consider closed sets and $F_{\sigma}$ sets.

$1^{\circ}$ Let $E$ be a bounded set inside the circle $C(O, R)$ of center $O$ and radius $R$; $d \mu$ being a positive mass distribution on $E$, let us consider both the potentials

\footnotetext{
${ }^{2}$ We recall that a subharmonic function in $C(O, R)$ is the sum of a harmonic function and a potential (2.2). For the general case, the reader will refer to [5] and [9].
} 


$$
\begin{aligned}
U^{\mu} & =\int \log |z-a| d \mu(a), \\
V^{\mu} & =\int \log \left|\frac{R(z-a)}{R^{2}-\bar{a} z}\right| d \mu(a) .
\end{aligned}
$$

The upper bound of the positive masses that $E$ can bear under the condition $V^{\mu} \geqq-1$ on $E$ is called the capacity of $E$ relative to $C(O, R)$.

A different notion will be obtained by considering the potential $U^{\mu}$ defined in the whole plane. Let us call $M_{\mu}$ the maximum of $U^{\mu}$ on $E$, for a mass distribution on $E$ of value 1 ; let $\gamma$ be the lower bound of $M_{\mu}$; then $C(E)=e^{-1 / \gamma}$ is called the logarithmic capacity of $E$.

It is easy to prove that both of the above defined measures of capacity are simultaneously vanishing.

A mass distribution will be called rational if it is composed of a finite number of punctual masses of rational values. To define the capacity $C(E)$ we may consider only rational distributions on $E$ [3 and 4]; in particular, on a given closed and bounded set $E$, of vanishing logarithmic cpacity, it is possible, for every $p>0$, to define a rational mass distribution of total value $\mu=1$, such that $U^{\mu} \leqq-p$ on $E$.

$2^{\circ} E=\sum_{i=1}^{\infty} E_{i}$, for bounded and closed $E_{i}$, and $C\left(E_{i}\right)=0$ yield $C(E)=0$.

Consequently, if $E$ is closed and unbounded, we say that $E$ is of vanishing capacity if, and only if, the intersection of $E$ with $C(O, R)$ is of vanishing capacity for every $R$. So in the whole plane, the point $\infty$ is a set of vanishing capacity.

When $u$ is a discontinuous function, we define its $u p p e r$ regularisation $u^{*}=$ reg. sup. $u$ by the following conditions $[7,8]$ :

(i) $u^{*} \geqq u$.

(ii) $u^{*}$ is upper semi-continuous.

(iii) $u^{*}$ is the smallest function satisfying (i) and (ii).

We may say that $u^{*}$ is the smallest upper semi-continuous majorant of $u$, or that $u^{*}$ is at every point equal to the Baire's maximum of $u$.

Let us recall some properties of upper-bounded sequences of potentials and subharmonic functions $u_{n}<M$.

[a $\mathrm{a}_{1}$ Let $u=\lim \sup u_{n}$ and $u^{*}=$ reg. sup. $u$. Then $u^{*}$ is a subharmonic function [6 and 7].

[a $]$ If $u_{n}^{r}(z)=(1 / 2 \pi) \int_{0}^{2 \pi} u_{n}\left(z+r e^{i \theta}\right) d \theta, u^{*}$ may be obtained by the following process: $u^{*}(z)=\lim _{r=0}\left[\lim \sup _{n} u_{n}^{r}(z)\right]$.

$\left[\mathrm{a}_{3}\right]$ We have $u \leqq u^{*}$ everywhere and $u=u^{*}$ except on a set of vanishing capacity. 
3. We shall now prove a very useful property of the sequences of polynomials. $^{3}$

THEOREM 2. Let $P_{n}(z)$ be a sequence of polynomials, with degree $P_{n} \leqq n ;$ the functions $u_{n}(z)=(1 / n) \log \left|P_{n}(z)\right|$ and their means $u_{n}^{r}(z)$ on the circumferences $C(z, r)$ have the following properties for $r>0$ :

A. $u_{n}^{r}(z)$ is a uniformly continuous family of functions of $z$, in the complex plane, for all $n, r$ belonging to an interval $r \geqq r_{0}>0$.

B. Let $\alpha_{r}=\lim \sup u_{n}^{r}(0)$ for a positive value of $r$. Then only three cases are possible:

(i) $\alpha_{r}=-\infty$. Then $u_{n}(z)$ converges uniformly to $-\infty$ in every bounded domain of the complex plane.

(ii) $\alpha_{r}=+\infty$. Then $u(z)=\lim \sup u_{n}(z)$ is $+\infty$ except on an $F_{\sigma}$ set of vanishing capacity.

(iii) $-\infty<\alpha_{r}<+\infty$. Then $u^{*}(z)=$ reg. sup. $u(z)$ is subharmonic in the plane; the set $u<u^{*}$ is an $F_{\sigma}$ of vanishing capacity.

The proof of $\mathrm{A}$ is easy. Let $\phi_{\rho}(r)$ be equal to $\log r$ if $r \geqq \rho$ and equal to $\log \rho$ if $0 \leqq r \leqq \rho: \phi_{\rho}(r)$ is a continuous function of $r$ and satisfies the following Lipschitz condition:

$$
\left|\phi_{\rho}(r)-\phi_{\rho}\left(r^{\prime}\right)\right| \leqq K_{\rho}\left|r-r^{\prime}\right| ; \quad K_{\rho}=1 / \rho .
$$

For every pair of points $z, a$, let us have

$$
\chi_{\rho}(z, a)=\phi_{\rho}(|z-a|) \text {. }
$$

If $P_{n}(z)=A_{n}\left(z-a_{1}\right) \cdots\left(z-a_{n}\right)$ and if $\mu_{n}$ is a distribution of $n^{\prime} \leqq n$ masses equal to $1 / n$, we get

$$
\begin{aligned}
& u_{n}(z)=\frac{1}{n} \log \left|P_{n}(z)\right|=\frac{1}{n} \log \left|A_{n}\right|+U^{\mu_{n}}, \\
& u_{n}^{r}(z)=\frac{1}{n} \log \left|A_{n}\right|+\int \chi_{r}(z, a) d \mu_{n}(a) .
\end{aligned}
$$

By (3.1) and (3.2), and $\int d \mu_{n} \leqq 1$, we find, for $r \geqq r_{0}>0$,

$$
\begin{aligned}
\int \chi_{r}\left(z_{1}, a\right) d \mu_{n}(a)-\int \chi_{r}\left(z_{2}, a\right) d \mu_{n}(a) & \\
& \leqq K_{r}\left|z_{1}-z_{2}\right| \leqq K_{r_{0}}\left|z_{1}-z_{2}\right|
\end{aligned}
$$

and, by (3.4),

$$
\left|u_{n}^{r}\left(z_{1}\right)-u_{n}^{r}\left(z_{2}\right)\right| \leqq K_{r_{0}}\left|z_{1}-z_{2}\right| .
$$

I In view of the other applications [see 7] we have once stated partially Theorem 2. 
The definition of $\phi_{\rho}(r)$ yields, for $\rho \geqq r_{0}, \rho^{\prime} \geqq r_{0},\left|\phi_{\rho}(r)-\phi_{\rho^{\prime}}(r)\right|$ $\leqq K_{r_{0}}\left|\rho-\rho^{\prime}\right|$,

$$
\left|\chi_{\rho}(z, a)-\chi_{\rho^{\prime}}(z, a)\right| \leqq K_{r_{0}}\left|\rho-\rho^{\prime}\right| \text {. }
$$

By (3.5) and (3.6) we get for $\rho>r_{0}, r>r_{0}$,

$$
\left|u_{n}^{\rho}\left(z_{1}\right)-u_{n}^{r}\left(z_{2}\right)\right|<K_{r_{0}}\left[|\rho-r|+\left|z_{1}-z_{2}\right|\right] .
$$

Part A of the theorem is therefore proved; (3.7) gives a very simple form of the Lipschitz condition.

Part B can be deduced from A and from the above recalled properties of the upper-bounded sequences of subharmonic functions.

(i) If $\alpha_{r}=-\infty$, then $u_{n}^{r}(0) \rightarrow-\infty$ and, by (3.7), $u_{n}^{r}(z)$ tends uniformly to $-\infty$ in every bounded domain; the majorization $u_{n}(z) \leqq u_{n}^{r}(z)$ yields that $u_{n}(z)$ also converges uniformly to $-\infty$; in case (i) we get $u(z)=u^{*}(z)=-\infty$.

(ii) If $\alpha_{r}=+\infty$, there exists a subsequence $u_{n_{p}}(z)$ such that $\lim u_{n_{p}}^{r}(0)=+\infty$. Let $\lambda_{p}=u_{n_{p}}^{r}(0)$, and $u_{p}^{l}(z)=\left(1 / \lambda_{p}\right) u_{n_{p}}(z) ;(3.7)$ shows that $u_{p}^{r}(z)$ tends to 1 for every $z$, and $r>0$.

(iii) If $\alpha_{r}$ is bounded, $u_{n}^{r}(z)$ is bounded and therefore $u(z)$ and $u^{*}(z)$ are upper bounded in every compact set (bounded and closed set) of the plane. Let us put

$$
u^{p}(z)=\sup _{n \geqq p} u_{n}(z)
$$

$u^{p}(z)$ is a nonincreasing sequence and $\lim _{p \rightarrow \infty} u^{p}(z)=u(z)$. On the other hand, $u_{n}(z)$ are continuous functions; $u^{p}(z)$ is lower semicontinuous and $u^{*}-u^{p}(z)$ is an upper semi-continuous function. The sets $E_{n, p}=E\left(u^{*}-u^{p} \geqq 1 / n\right)$ are therefore closed sets, and nondecreasing functions of $n$ and $p ; E=E\left(u^{*}-u>0\right)=\lim _{n, p, \infty} E_{n, p}=\sum_{n, p} E_{n, p}$ is therefore an $F_{\sigma}$ set; $E=E\left(u<u^{*}\right)$ is, according to property [a $\left.\mathrm{a}_{3}\right]$, an $F_{\sigma}$ of vanishing capacity.

By property $\left[a_{2}\right]$ we get

$$
u^{\prime *}(z)=\text { reg. sup. }\left[\lim \sup u_{n}(z)\right]=\lim _{r=0}\left[\lim \sup u_{n}(z)\right]=1 \text {. }
$$

The set $E(u<\infty)$ is a subset of $E\left(u^{\prime}=0\right)$ and $E\left(u^{\prime}=0\right) \subset E\left(x^{\prime}<1\right)$ $=E\left(u^{\prime}<u^{\prime *}\right)$. The set $E(u<\infty)$ is therefore of vanishing capacity. It is also an $F_{\sigma}$ (see (iii)). Theorem 2 is proved.

Now, we shall establish a reciprocal proposition of $B$, case (ii).

THEOREM 3. If the set $E$ in the complex plane is contained in an $F_{\sigma}$ of vanishing capacity, there exists a sequence $P_{n}(z)$ of polynomials with degree $P_{n} \leqq n$, such that $u(z)=\lim \sup (1 / n) \log \left|P_{n}(z)\right|$ is equal to 
$+\infty$ except on a set $F_{\sigma}^{\prime}$ of vanishing capacity containing the given set $E$.

Proof. By the hypothesis we get $E \subset F_{\sigma}=\sum E_{n} ; E_{n}$ is a closed set; when $E_{n}$ is unbounded let us call $E_{n, p}$ the intersection of $E_{n}$ with $C(O, n)$,

$$
E \subset \sum_{n, p} E_{n, p}=\lim \varepsilon_{k} \text { with } \varepsilon_{k}=\sum_{n+p=k} E_{n, p} .
$$

$\varepsilon_{k}$ is closed and bounded; thus there exists a rational mass distribution of value 1 on $\varepsilon_{k}$ such that on $\varepsilon_{k}$,

$$
u^{\mu_{k}}=\sum \frac{1}{N_{k}} \log \left|z-a_{i, k}\right| \alpha_{i, k} \leqq-K .
$$

$\sum_{i} \alpha_{i, k}=N_{k} ; \alpha_{i, k}, N_{k}$ are positive integers. Let us have

$$
P_{k}(z)=e^{k} \Pi\left(z-a_{i, k}\right)^{\alpha_{i, k},} u(z)=\lim \sup _{k} \frac{1}{k} \log \left|P_{k}(z)\right|=u^{\mu_{k}}+k .
$$

$u(z)$ is less than or equal to 1 on $F_{\sigma}=\sum_{p} \varepsilon_{p}$; on the other hand $u_{k}^{r}(0) \rightarrow+\infty$ when $k \rightarrow+\infty$. Theorem 2 , case (ii), yields $u(z)=+\infty$ except on an $F_{\sigma}^{\prime} \supset F_{\sigma} \supset E$. Theorem 3 is proved.

4. To prove Theorem $1_{\mathrm{a}}$, let us now consider the given class $\left[S_{a, b}\right]$ of substitutions (1.1) (we recall that $S_{00}$ is excepted from $\left[S_{a, b}\right]$ ). Let $E(\beta)=\varepsilon$ be the image set of $\left[S_{a, b}\right]$ in the complex plane of the variable $\beta=b / a$. We may consider a new class $\left[\sigma_{a, b}\right]$ of substitutions defined as follows.

$$
S_{a b} \in\left[\sigma_{a, b}\right] \text { if } a=1 \text { and } b=\beta / \alpha \in \mathcal{E} .
$$

The image set of $\left[\sigma_{a, b}\right]$ in the $\beta$-plane is also $\varepsilon$, and therefore $F_{a b}(t)$ is a convergent power series for $a=1, b \in \varepsilon$,

$$
F_{a b}(t)=\sum_{n=0}^{\infty} t^{n} \sum_{j=0}^{j=n} a_{n-j, j} b^{j}=\sum t^{n} P_{n}(b) .
$$

$P_{n}(z)=\sum_{j=0}^{j=n} a_{n-j, j} z^{j}$ is of degree less than or equal to $n$.

For $z \in \varepsilon$, the radius $\rho$ of convergence of (4.2) is nonvanishing.

$$
-\log \rho=\lim \sup \frac{1}{n} \log \left|P_{n}(z)\right|<+\infty \quad \text { for } z \in \mathcal{E} .
$$

By the hypothesis, $\varepsilon$ is not a subset of an $F_{\sigma}$ set of vanishing capacity. Let us consider the sequence

$$
u_{n}(z)=\frac{1}{n} \log \left|P_{n}(z)\right|
$$


and apply Theorem 2. (4.3) is exclusive of the case (ii); $u_{n}^{r}(z)$ are in consequence uniformly bounded in the circle $C(O, 2 r)$; in $C(O, r)$ we get a majorization $u_{n}(z) \leqq u_{n}^{r}(z) \leqq \log M ; \quad\left|P_{n}(z)\right| \leqq M^{n}$.

Cauchy's formula yields

$$
\left|a_{n-j, j}\right| \leqq M^{n} r^{-j}
$$

$F=\sum_{i, j} a_{i j} x^{i} y^{j}$ is therefore majorized by

$$
\bar{F}=\sum_{i, j}\left|a_{i j} x^{i} y^{j}\right| \leqq M^{i+j}\left|x^{i}\right|\left|y^{j}\right|
$$

which converges for $|x|<1 / M,|y| \leqq r / M$. Theorem $1_{\mathrm{a}}$ is proved.

Proof of Theorem $1_{b}$. We suppose now that the class $\left[S_{a, b}\right]$ making $F_{a b}(t)$ convergent corresponds ( $S_{00}$ excepted) to an image set $\varepsilon=E(\beta)$ which is a subset of an $F_{\sigma}$ of vanishing capacity. We shall prove that $\left[S_{a, b}\right]$ is not a normal class of substitutions (1.1).

By Theorem 3 , there exists a sequence of polynomials $P_{n}(z)$ $=\sum_{j=0}^{j=n} a_{n-j, j} z^{j}$ such that if $u_{n}(z)=(1 / n) \log \left|P_{n}(z)\right|$, we have

$$
\lim \dot{u}_{n}^{r}(0)=\infty \text {. }
$$

The sequence of polynomials $P_{n}(z)$ belongs to case (ii) of Theorem 2.

Let us put $F=\sum_{i, j} a_{i j} x^{i} y^{j} . F$ becomes convergent by the substitutions $S_{a b} \in\left[S_{a, b}\right]$.

On the other hand, if $F$ were an analytic function of $x, y$ for $|x|$ $\leqq r,|y| \leqq r, M_{1}$ being the maximum of $|F(x, y)|$ for $|x|=|y|=r$, we could write, by Cauchy's formula,

$$
\left|a_{i j}\right| \leqq M_{1} r^{-(i+j)} \text {. }
$$

$\bar{F}=\sum_{i, j}\left|a_{i j} x^{i} y^{j}\right|$ is majorized by the series $M \sum|x / r|{ }^{i}|y / r|^{i}$ which converges for $|x|<r,|y|<r$, or for $|x| \leqq \rho,|y| \leqq \rho$, and $\rho<r$.

Reciprocally, if $\bar{F}$ converges for $|x| \leqq r,|y| \leqq r$, and $\bar{F}(r, r)=M_{2}$, we get a majorization $\left|a_{i j}\right| \leqq M_{2} r^{-(i+j)}$ of the same form. It is therefore sufficient to prove that (4.6) and (4.5) are in contradiction; (4.6) yields, for $|z| \leqq 1,\left|P_{n}(z)\right| \leqq \sum_{i+j=n}\left|a_{i j}\right| \leqq(n+1) M_{1} . u_{n}(z)=(1 / n) \log$ $\left|P_{n}(z)\right|$ is therefore upper bounded for $|z|<1 ; u_{n}^{r}(0)$ is upper bounded for $0<r<1$; the contradiction with (4.5) and, as a consequence, Theorem $1_{b}$, is proved.

As a consequence of the preceding results, we may remark that two alternatives only are possible concerning the class $\sigma(F)$ of substitutions (1.1) which transform a formal development into a convergent series $F_{a b}(t)$ : either every $S_{a b}$ belongs to $\sigma(F)$, or the image set of $\sigma(F)$ is an $F_{\sigma}$ set of vanishing capacity. 
In view of applications, let us remark that every enumerable set (for example the set of rational or algebraic numbers $a, b$ ) of substitutions $S_{a b}$ is non-normal, that is to say, it is impossible, without using particular hypothesis on the $a_{i j}$, to deduce the convergence of $\bar{F}$ (except for $x=y=0$ ) and the analy ticity of $F$ from the convergence of the series $F_{a b}(t)$.

\title{
BIBLIOGRAPHY
}

1. M. A. Zorn, Bull. Amer. Math. Soc. vol. 53 (1947) pp. 791-792

2. Rimhak Ree, Bull. Amer. Math. Soc. vol. 55 (1949) pp. 575-576.

3. Evans, Monatshefte für Mathematik vol. 43 (1936) pp. 419-424.

4. G. P6lya and G. Szegö, J. Reine Angew. Math. vol. 165 (1931) pp. 4-49.

5. T. Rado, Subharmonic functions, Ergebnisse der Mathematik und ihre Grenzgebiete, vol. $5,1,1937$.

6. M. Brelot, C. R. Acad. Sci. Paris vol. 207 (1938) p. 836.

7. P. Lelong, C. R. Acad. Sci. Paris vol. 210 (1940) p. 471.

8. - Ann. Ecole Norm. vol. 58 (1941) pp. 83-177.

9. H. Cartan, Bull. Soc. Math. France vol. 73 (1945) pp. 74-106.

FACULte DES SCIENCES DE LiLle

\section{NOTE ON A SERIES OF PRODUCTS OF THREE LEGENDRE POLYNOMIALS}

\author{
JOHN P. VINTI
}

Let $x, y$, and $z$ be real variables and $P_{l}$ the Legendre polynomial of order $l$. In this note we shall prove the following theorem:

$$
\begin{aligned}
& \sum_{l=0}^{\infty}\left(l+\frac{1}{2}\right) P_{l}(x) P_{l}(y) P_{l}(z)=\pi^{-1} g^{-1 / 2} \quad(g>0) \\
& =0 \quad(g<0) \\
& (-1<x<1,-1<y<1,-1<z<1) \text {, }
\end{aligned}
$$

where

$$
g(x, y, z) \equiv 1-x^{2}-y^{2}-z^{2}+2 x y z .
$$

Furthermore, if $T_{+}$be the region of $x, y, z$ space, as above delimited, for which $g>0$, and $T$ - be the region for which $g<0$, the convergence is uniform with respect to any one of the variables $x, y$, or $z$, taken singly, that is, throughout any closed interval along which only one of the variables changes and which is interior either to $T_{+}$or to $T_{-}$.

Received by the editors November 2, 1949. 